02

\title{
Разброс в измерениях времени жизни отрицательных ионов как следствие их адсорбции на стенках камеры ионизации
}

\author{
(C) В.Г. ЛУкин, О.Г. Хвостенко, Г.М. ТУймедов \\ Институт фоиики молекул и кристаллов УНЦ РАН, \\ 450075 Уфра, Россия \\ e-mail: lukin@anrb.ru
}

(Поступило в Редакцию 29 февраля 2016 г. В окончательной редакции 20 июня 2016 г.)

\begin{abstract}
Измерено время вытягивания отрицательных ионов из камеры ионизации статического масс-спектрометра как величина, значительно превышающая время их свободного выхода из камеры. Установлено, что аномально большое время вытягивания ионов обусловлено их адсорбцией на стенках камеры ионизации, в результате чего их перемещение в трубу анализатора задерживается. Показано, что отрицательные ионы, образующиеся первоначально как временноживущие относительно автоотщепления добавочного электрона, в результате адсорбции стабилизуются до вечноживущих, и последующий вклад вечноживущих ионов в полный ионный поток, достигающий систему регистрации, искажает результаты измерений времени жизни ионов. Показано, что часть адсорбированных ионов гибнет за счет нейтрализации в результате туннелирования добавочного электрона в поверхность. Вероятность туннелирования возрастает с ростом температуры, и в итоге температурная зависимости времени жизни ионов также искажается.
\end{abstract}

DOI: 10.21883/JTF.2017.07.44666.1786

\section{Введение}

При экспериментальном измерении среднего времени время жизни $\left(\tau_{a}\right)$ отрицательных молекулярных ионов $\left({ }^{a} \mathrm{M}^{-}\right)$, образующихся при резонансном захвате электронов (РЗЭ) молекулами (энергии электронов составляют 0-15 eV), часто возникает проблема большого разброса регистрируемых величин $\tau_{a}$. Этот разброс возникает, в частности, тогда, когда величины $\tau_{a}$ измеряются с применением различной приборной техники. Например, в обзоре [1] приведены результаты измерений, полученные на ионно-циклотронных и времяпролетных масс-спектрометрах: для $\mathrm{SF}_{6}^{-} \tau_{a}=10,25$ и $68 \mu \mathrm{s}$; для $\mathrm{C}_{6} \mathrm{H}_{5} \mathrm{NO}_{2}^{-}-18,40,47$ и $49 \mu \mathrm{s}$; для $\mathrm{C}_{6} \mathrm{~F}_{6}^{-}-12$ и $13 \mu \mathrm{s}$. Однако разброс имеет место и при проведении исследований на приборе одного типа. Например, для вышеперечисленных ионов имеется аналогичный разброс в измерениях $\tau_{a}$, выполненных с использованием статического масс-спектрометра [2]. Для $\mathrm{SF}_{6}^{-}$в разное время были получены величины $\tau_{a}$ от 70 до $140 \mu \mathrm{s}$, для $\mathrm{C}_{6} \mathrm{H}_{5} \mathrm{NO}_{2}^{-}$от 58 до $90 \mu \mathrm{s}$, для $\mathrm{C}_{6} \mathrm{~F}_{6}^{-} 35 \mu \mathrm{s}$, для изученного в настоящей работе дурохинона (DQ) от 137 до $191 \mu \mathrm{s}$.

Общепринятое объяснение экспериментального разброса измеренных $\tau_{a}$ состоит в том, что временноживущие ${ }^{a} \mathrm{M}^{-}$образуются в камере ионизации (IC) из исследуемых молекул с разной заселенностью колебательных уровней, которая зависит от температуры [3]. В результате распад ионов ${ }^{a} \mathrm{M}^{-}$приобретает многоэкспоненциальный характер, а измеряемое $\tau_{a}$ становится функцией „временного окна“ $\Delta t-$ времени, протекающего от момента образования ионов в IC до момента их регистрации первым динодом вторичного электронного умножителя - secondary electronic multiplier (SEM). Чем больше $\Delta t$, тем меньше короткоживущих ионов долетает до системы регистрации, и, следовательно, $\tau_{a}$ измеряется как бо́льшая величина. Отсюда видно, что в случае справедливости этой концепции должна существовать прямая корреляция между $\tau_{a}$ и $\Delta t$. Для ее проверки в настоящей работе с использованием одного и того же прибора - модифицированного массспектрометра МИ-1201В были одновременно измерены обе величины: $\tau_{a}$ и $\Delta t$. Также для выявления других возможных причин разброса $\tau_{a}$ были проведены измерения обеих величин $\left(\tau_{a}\right.$ и $\left.\Delta t\right)$, но при разных настройках прибора и с варьированием различных условий эксперимента.

При разработке методики, примененной в настоящей работе, учитывалось, что временное окно $\Delta t$ определяется следующим выражением:

$$
\Delta t=t_{\mathrm{ext}}+t_{f}+t_{t p}
$$

где $t_{\mathrm{ext}}-$ время вытягивания ионов из $\mathrm{IC}, t_{f}-$ время пролета иона по трубе масс-спектрометра от IC ионизации до SEM и $t_{t p}=20 \mu \mathrm{s}-$ время переходных процессов в оптронной паре (свето-/фотодиод) электронного реле, использованного при измерениях. Величина $t_{f}=18.5 \mu \mathrm{s}$ для $\mathrm{DQ}^{-}$была измерена ранее в [2] и, так же как и величина $t_{t p}$, принималась за константу при рабочем ускоряющем напряжении, равном $4 \mathrm{kV}$. Таким образом, ввиду того, что два слагаемых $t_{f}$ и $t_{t p}$ вышеприведенной суммы (1) являются константами, искомая корреляция была изучена в настоящей работе как корреляция между $\tau_{a}$ и $t_{\mathrm{ext}}$. Измерения $\tau_{a}$ и $\Delta t \mathrm{c}$ последующими вычислениями величины $t_{\mathrm{ext}}$ на основе уравнения (1) были выполнены как при стандартных экспериментальных условиях, так и при их изменениях. 


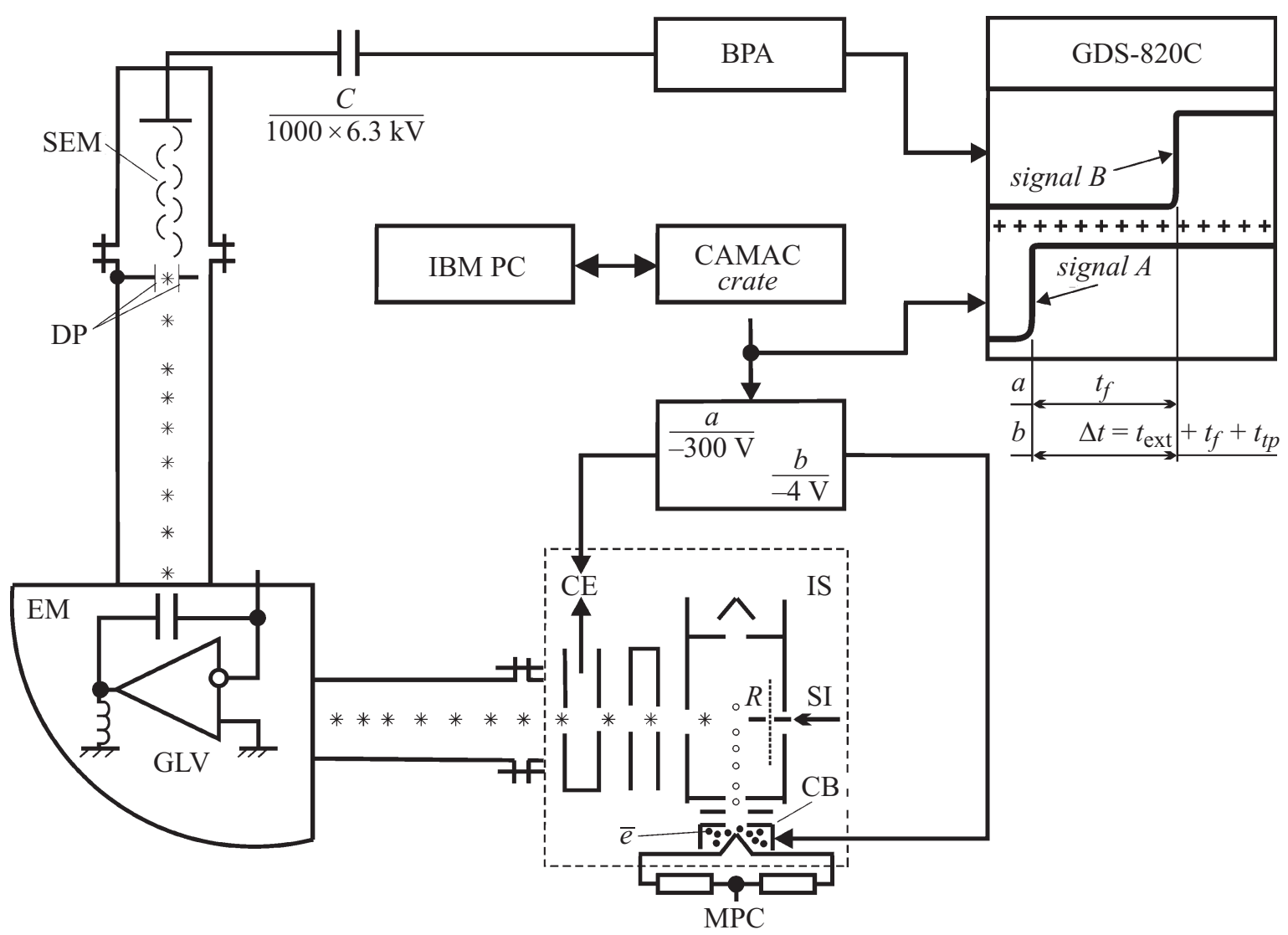

Рис. 1. Функциональная схема модернизированного масс-спектрометра МИ-1201В с двуканальным цифровым осциллографом GDS-820C. SI - напуск вещества, IS - источник ионов, $R$ - выталкивающий электрод, СЕ - корректирующий электрод, EM - электромагнит, GLV - генератор линейного напряжения для развертки напряженности магнитного поля анализатора, ВРА - широкополосный импульсный усилитель, МРС - средняя точка катода. Другие обозначения, приведенные на рисунке, см. в тексте.

\section{Экспериментальные методики}

Исследования настоящей работы проведены на серийном масс-спектрометре МИ-1201В (рис. 1) с магнитным анализатором секторного типа $[4,5]$, переоборудованном для регистрации отрицательных ионов (ОИ) в режиме резонансного захвата моноэнергетичных электронов молекулами, когда энергия электронов варьирует от нуля до $15 \mathrm{eV}$ [6]. Также прибор был укомплектован осциллографом для измерения $t_{f}$ и $\Delta t[2,7]$. Высокий вакуум $\left(\sim 10^{-6} \mathrm{~Pa}\right)$ в камере анализатора прибора создается двумя паромасляными диффузионными насосами и измеряется магнитоионизационными датчиками, расположенными в области насосов. В системе регистрации первый динод SEM заземлен, что обеспечивает регистрацию ОИ и нейтральных молекул, образующихся автоотщеплением добавочного электрона ОИ, как частиц с одинаковой энергией. Прибор сопряжен с IBM РC в стандарте КАМАК с применением интерфейсного устройства АПЭК 5.0, произведенного НТО АН СССР (Черноголовка) [8]. Программное обеспечение написано на языке высокого уровня Visual Basic. Исследуемое вещество напускается в IC через „гребенку“ или пря- мой ввод [9]. В процессе работы прибора электроны, испускаемые $\mathrm{W}$-катодом, взаимодействуют с парами вещества и образуют ОИ. Последние вытягиваются из IC, ускоряются, разделяются по массовым числам $(\mathrm{m} / \mathrm{z})$ и регистрируются. Процедура исследования в режиме реального времени проводится в два этапа, поскольку масс-спектр резонансного захвата электронов молекулами представляет собой набор кривых эффективного выхода (КЭВ) в трехмерном измерении: массовое число-интенсивность-энергия электронов. Сначала в заданном диапазоне массовых чисел записывается массспектр ОИ с одновременным сканированием по энергии электронов от 0 до $15 \mathrm{eV}$, затем записываются КЭВ ионов для каждого $m / z$.

Методика измерения среднего времени жизни ОИ относительно автоотщепления электрона основана на выделении нейтральных молекул $N^{0}$ из полного пучка $N$ и соответствующей записи для них КЭВ $[10,11]$. Выделение $N^{0}$ происходит в электрическом поле плоского конденсатора, подачей на его пластины, установленные перед SEM, отрицательного потенциала $(1-1.5) \mathrm{kV}$. Автоотщепление добавочного электрона ОИ носит статистический характер, подобно закону радиоактивного 
Результаты измерений времени жизни $\tau_{a}$ и времени вытягивания $t_{\mathrm{ext}}$ из камеры ионизации отрицательных ионов DQ в зависимости от даты проведения исследований

\begin{tabular}{|c|c|c|c|c|c|c|c|}
\hline \multirow{2}{*}{$\begin{array}{c}\text { Измеряемая } \\
\text { величина, } \\
\mu \mathrm{s}\end{array}$} & \multicolumn{6}{|c|}{$\begin{array}{l}\text { Символическое обозначение } \\
\text { даты проведения измерений }\end{array}$} & \multirow{2}{*}{$\begin{array}{c}\text { Диапазон } \\
\text { значений, } \\
\mu \mathrm{s}\end{array}$} \\
\hline & 1 & 2 & 3 & 4 & 5 & 6 & \\
\hline$\tau_{a}$ & 191 & 191 & 153 & 146 & 143 & 137 & $137-191$ \\
\hline$t_{\mathrm{ext}}$ & 16 & 110 & 6.5 & 15 & 69 & 23.5 & $6.5-110$ \\
\hline
\end{tabular}

распада. Соответственно часть первоначального общего количества ионов $N$ за время пролета $t$ от массанализатора до первого динода SEM испытает акт автоотщепления, превращаясь в нейтральные молекулы. Среднее время жизни ОИ $\tau_{a}$ вычисляется по формуле

$$
\tau_{a}=t / \ln \left[\left(N-N^{0}\right) / N\right]
$$

где $N=N^{-}+N^{0}$ - число ионов в пучке, которые в момент $t=0$ входят в пространство, свободное от электрических и магнитных полей; $N^{-}$- число ионов, сохранившихся за время $t ; \tau_{a}-$ среднее время жизни ОИ.

Методики измерения $t_{f}$ и $\Delta t$ с использованием двухлучевого осциллографа серии GDS-820C [12] подобны. Различия в том, что при измерении $t_{f}$ запирается/отпирается ионный пучок подачей/снятием отрицательного потенциала $\sim 300 \mathrm{~V}$ на корректирующий электрод - correcting electrode (CE) (рис. 1), а при измерении $\Delta t$ запирается/отпирается электронный пучок подачей отрицательного потенциала $\sim 4 \mathrm{~V}$ на модулятор - катодную коробочку cathode box (CВ) (рис. 1). Кроме того, управление процедурой измерения $\Delta t$ осуществляется через электронное реле [7], поскольку электронно-оптическая система источника ионов находится под ускоряющим ОИ потенциалом. В обеих методиках осциллограф используется в ждущем режиме с синхронизацией по фронту, когда запуск развертки возможен только при наличии запускающего сигнала, амплитуда которого должна быть больше уровня запуска развертки. Этот сигнал от IBM РC через КАМАК подается на один из каналов GDS-820C, что синхронно запускает развертку обоих каналов с отображением на экране осциллографа. На второй канал подается сигнал широкополосного импульсного усилителя, подключенного через разделительный конденсатор к SEM, который после поступления пучка ОИ на первый динод умножителя также отображается осциллографом. В зависимости от выполняемой процедуры разностью между этими двумя сигналами определяется либо $t_{f}$, либо $\Delta t$. Затем вычисляется $t_{\mathrm{ext}}$ по формуле, вытекающей из вышеприведенного уравнения (1):

$$
t_{\mathrm{ext}}=\Delta t-\left(t_{f}+t_{t p}\right) \text {. }
$$

\section{Экспериментальные результаты}

Проведены измерения $\tau_{a}$ и $t_{\text {ext }}$ для ионов $\mathrm{DQ}^{-}$в разное время при одних и тех же стандартных условиях эксперимента, при номинальных потенциалах на линзах ионного источника. Результаты представлены в таблице, откуда видно, что обе величины весьма заметно меняются в зависимости от даты измерений. Величина $\tau_{a}$ ионов $\mathrm{DQ}^{-}$варьирует в интервале $191-137 \mu \mathrm{s}$, а величина $t_{\mathrm{ext}}$ колеблется в интервале 6.5-110 $\mu$ s. Следует отметить, что $\tau_{a}$ и $t_{\text {ext }}$ не коррелируют друг с другом, как это можно было ожидать с учетом концепции многоэкспоненциальности распада.

Обе величины $\tau_{a}$ и $t_{\text {ext }}$, равно как и интенсивность ионов $\mathrm{DQ}^{-}$, были измерены как функции количества DQ, напускаемого в IC (рис. 2), с целью изучения влияния вакуума на первые две величины,

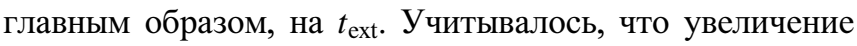
напуска вещества в камеру ионизации приводит к ухудшению вакуума. Из рис. 2, $b$ видно, что время вытягивания $t_{\text {ext }}$ при увеличении напуска и, следовательно, при ухудшении вакуума резко падает. Время жизни $\tau_{a}$ при этом демонстрирует осцилляцию около некоторой постоянной величины $\sim 170 \mu \mathrm{s}$ (рис. $2, a)$ и, так же, как и в вышеописанном случае измерений, проведенных в разное время, не коррелирует с $t_{\text {ext. }}$ Сам факт увеличения напуска вещества виден на графике интенсивности соответствующих молекулярных ионов $\mathrm{DQ}^{-}$(рис. 2,c), которая совершенно закономерно возрастает при увеличении числа оборотов вентиля напуска.

Экспериментально изучена температурная зависимость всех трех величин - $\tau_{a}, t_{\text {ext }}$ и интенсивности ионов $\mathrm{DQ}^{-}$, которая показана на рис. 3. Из рис. 3, $a$ видно, что с ростом температуры IC от 333 до $473 \mathrm{~K}$ величина $\tau_{a}$ значительно уменьшается: от 152.8 до $81 \mu \mathrm{s}$. В то же время наблюдается рост времени вытягивания с 6.5 до $31.8 \mu \mathrm{s}$. Обращает на себя внимание тот факт, что при увеличении температуры IC интенсивность ионного пучка уменьшается в 2.3 раза, с $9 \cdot 10^{4}$ до $4 \cdot 10^{4} \mathrm{pulses} / \mathrm{s}$.

\section{Обсуждение экспериментальных результатов}

Измерения, выполненные при одинаковых условиях, но в разное время (см. таблицу), показывают, что измеренные величины и $\tau_{a}$, и $t_{\mathrm{ext}}$ ионов $\mathrm{DQ}^{-}$меняются в зависимости от даты проведения исследований, демонстрируя явный разброс. Полученный разброс обеих величин труднообъясним, поскольку в данном случае никакие экспериментальные условия, включая температуру, не менялись. Также из полученных данных, как отмечалось выше, видно, что отсутствует ожидаемая корреляция между $\tau_{a}$ и $t_{\text {ext }}$, вытекающая из концепции температурной многоэкспоненциальности. Поскольку ожидаемая корреляция не обнаруживается, необходимо сделать вывод, что различная температурная 

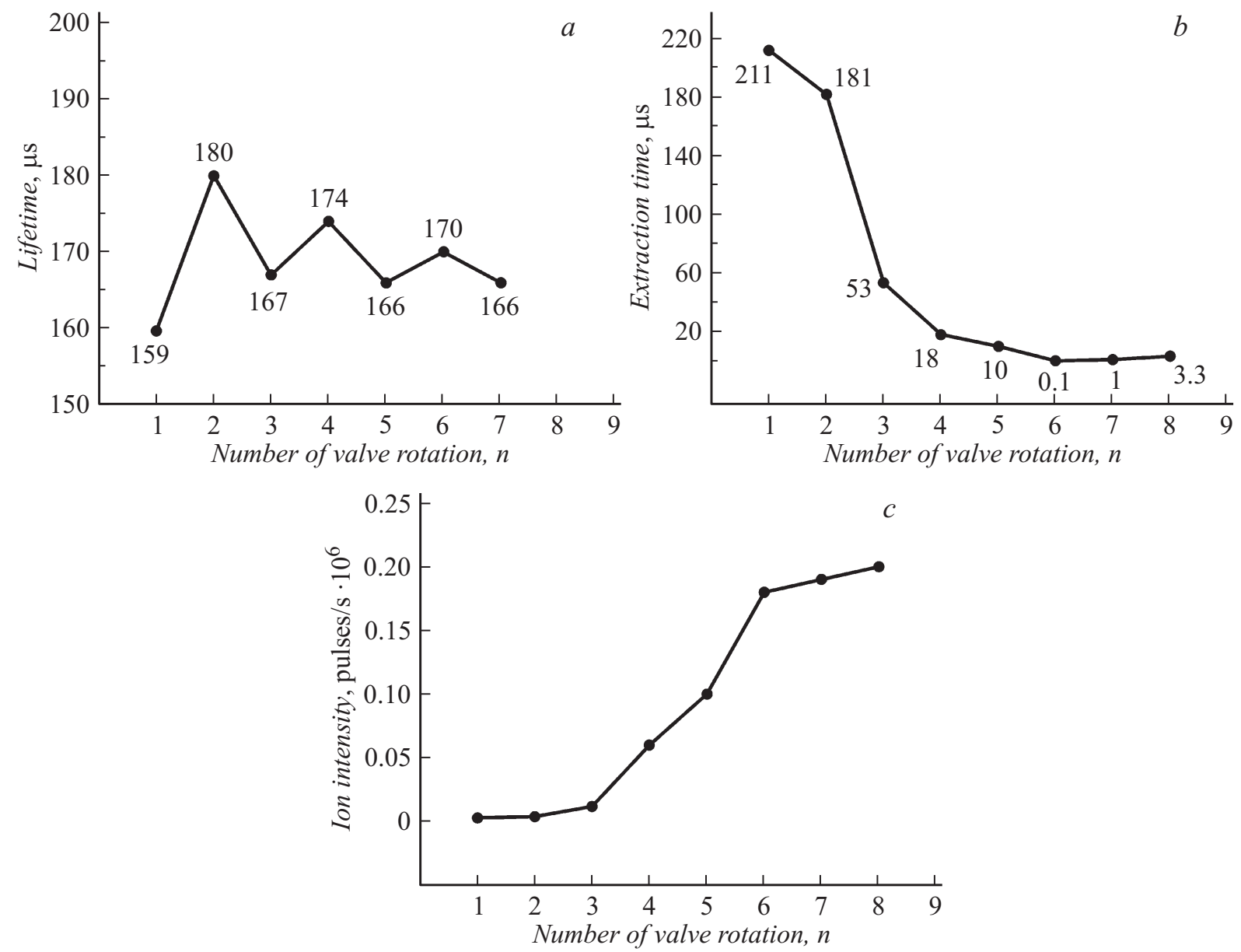

Рис. 2. Время жизни $(a)$, время вытягивания из камеры ионизации $(b)$ и интенсивность $(c)$ отрицательных ионов DQ в зависимости от количества вещества, напускаемого в камеру ионизации; $n-$ число оборотов вентиля напуска.

заселенность исходных молекул является не единственной причиной разброса в измеренных величинах $\tau_{a}$ и в него вносят свой вклад другие эффекты. Для выяснения происхождения этих эффектов в настоящей работе была проанализирована величина $t_{\mathrm{ext}} \mathrm{c}$ точки зрения ее абсолютных значений.

Величины $t_{\mathrm{ext}}$ ионов $\mathrm{DQ}^{-}$, приведенные в таблице, составляют десятки $\mu \mathrm{s}$, что значительно превышает время свободного выхода иона $\mathrm{DQ}^{-}$из камеры ионизации, которое составляет $\sim 4 \mu$ s для номинальных потенциалов на ее линзах. Очевидно, что ионы, образовавшиеся в камере ионизации, задерживаются там, прежде чем ее покинуть. Тому могут быть три возможные причины:

1) удлинение траектории ионов из-за упругих столкновений в IC с молекулами остаточного газа, столкновений, которые, возможно, удлиняют траекторию их движения в процессе выхода из IC;

2) блокирование ионов в камере ионизации объемным зарядом;

3) адсорбция ионов на поверхности стенок IC.

Чтобы проанализировать возможность задержки ионов в IC за счет столкновений ионов с молекулами остаточного газа, была вычислена длина свободного пробега $(l)$ молекул в IC при давлении $P \ll 10^{4} \mathrm{~Pa}$, в котором проводились эксперименты. Использовалось выражение, приведенное в работе [13]:

$$
l=[(T-273) / 273] \cdot(1 / P),
$$

где $l$ - рассчитана в $\mathrm{m}, T$ - температура в $\mathrm{K}, P$ давление в Ра. Было получено $l \sim 22 \mathrm{~m}$, что указывает на малую вероятность столкновения ионов с остаточным газом ( $\sim 0.05 \%$ от всех ионов). Это означает, что задержку ионов в IC объяснить увеличением длины их траектории по причине недостаточно высокого вакуума невозможно. Этот же вывод прямо подтверждается экспериментом, в котором величина $t_{\mathrm{ext}}$ была измерена как функция количества DQ, напускаемого в IC (рис. 2). Как уже упоминалось выше, увеличение напуска вещества в IC заведомо ухудшает вакуум. В то же время, как видно из рис. $2, b$, при увеличении напуска $t_{\mathrm{ext}}$ не растет, как это было бы в случае задержки иона из-за столкновений, a, наоборот, падает. Таким образом, эксперимент с увеличением напуска еще раз подтверждает вывод, полученный на основе оценки длины свободного пробега, что задержка ионов в IC с параметрами вакуума не связана. 

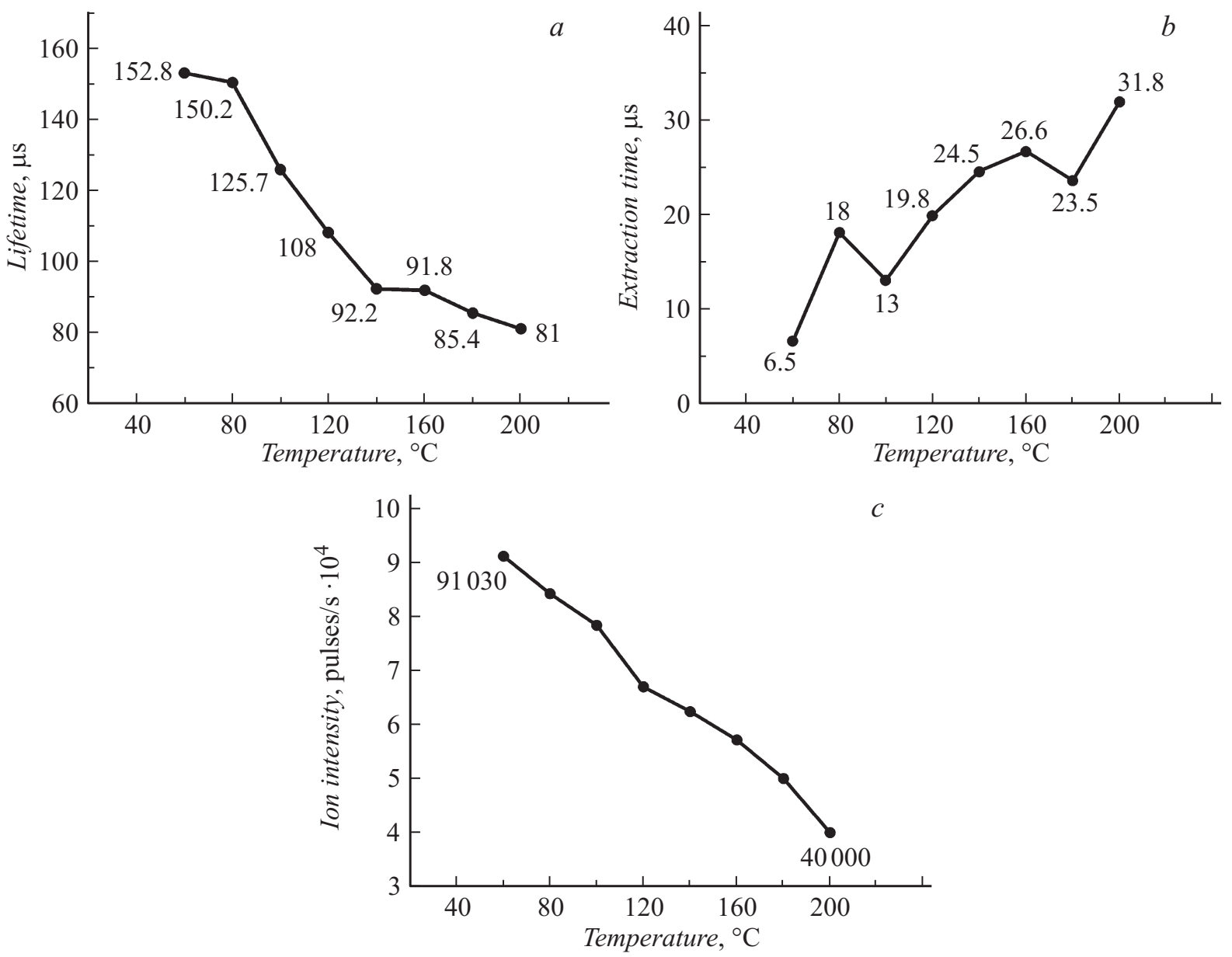

Рис. 3. Время жизни $(a)$, время вытягивания из камеры ионизации $(b)$ и интенсивность $(c)$ отрицательных ионов дурохинона в зависимости от температуры камеры ионизации.

Эксперимент с увеличением напуска исследуемого вещества (в данном случае - дурохинона) в камеру ионизации не позволяет подтвердить и вторую из вышеперечисленных возможную причину задержки ионов в ней, а именно воздействие объемного заряда, который мог бы создавать ионную блокаду. Очевидно, что при увеличении напуска, количество ионов, образующихся в IC, возрастает. На это указывает рост интенсивности ионов при увеличении напуска (рис. 2, c). Очевидно, что и объемный заряд, если таковой вообще образуется, в этом случае также должен увеличиваться. И если бы объемный заряд был причиной задержки иона в IC, время вытягивания $t_{\text {ext }}$ при увеличении напуска также бы возрастало. Однако роста $t_{\mathrm{ext}}$, как уже отмечалось выше, не происходит, и, следовательно, вторая из вышеперечисленных возможная причина задержки ионов в IC также должна быть отвергнута. Следует отметить, что непричастность объемного заряда к проблеме задержки ионов в IC в принципе вполне объяснима, поскольку в масс-спектрометрии отрицательных ионов РЗЭ образование объемного заряда очень мало вероятно (в отличие от масс-спектрометрии положительных ионов). Причина в том, что сечения образования отрицательных ионов в IC масс-спектрометра значительно меньше сечений образования положительных ионов - по меньшей мере на три порядка [14]. Кроме того, есть еще один фактор, который еще более минимизирует возможность возникновения объемного заряда отрицательных ионов в камере ионизации данного масс-спектрометра: это отрицательный потенциал выталкивающего электрода („, $R^{\text {‘ }}$ на рис. 1$)$, который форсирует продвижение образующихся отрицательных ионов из IC в трубу анализатора.

Учитывая рассмотрение первых двух причин, которые были вынужденно отвергнуты, следует прийти к выводу, что причиной задержки ионов в IC может быть только адсорбция ионов на ее стенках. Это означает, что ион присоединяется к проводящей поверхности IC за счет индуцированного заряда, создаваемого в этой поверхности самим ионом на глубине зеркального отображения (image potential), и только затем, через какое-то время $\left(t_{\text {ext }}\right)$, в результате десорбции под воздействием колебательных взаимодействий отрывается от нее и вытягивается из IC. Причем адсорбированный ион отделен от поверхности и соответственно от индуцированного заряда слоем молекул самого напускаемого вещества, который, как известно, всегда присутствует на стенках IC. 
И очевидно, что чем больше толщина $(L)$ молекулярного слоя, покрывающего проводящую поверхность стенки, тем слабее связь иона с ней и тем меньше времени он проведет на ней, прежде чем произойдет его десорбция.

В результате адсорбции на стенках IC временноживущие ионы $\left({ }^{a} \mathrm{M}^{-}\right)$подвергаются значительным изменениям. Изменения выражаются в том, что временноживущие ионы ${ }^{a} \mathrm{M}^{-}$трансформируются на поверхности в вечноживущие $\left({ }^{\infty} \mathrm{M}^{-}\right)$. Причина этого явления состоит в следующем. Общеизвестно, что теплопередача всегда происходит от тел с более высокой температурой к телам с более низкой. Поэтому при взаимодействии ионов со стенкой колебательная энергия иона передается стенке, а точнее - молекулярному слою, покрывающему стенку. Это происходит потому, что ионы образуются не на нулевом колебательном уровне, а на гораздо более высоком, в то время как молекулы слоя, отделяющего ион от поверхности, находятся по большей части на своем нулевом колебательном уровне. Другими словами, ион в определенном смысле является более „горячим“ объектом, чем прилегающие к нему молекулы, что приводит к передаче колебательной энергии именно от иона к стенке. Как результат, колебательная энергия иона уменьшается, и он оказывается ниже того колебательного уровня, на котором образовался. С другой стороны, по нашим данным, ион $\mathrm{DQ}^{-}$образуется в квартетном состоянии посредством интеркомбинационной конверсии из материнского иона-дублета за счет безызлучательного перехода с инверсией электронного спина в точке пересечения поверхностей потенциальной энергии „исходного“ (материнского) иона-дублета и иона-квартета [15-18]. Переход иона-квартета на более низкий колебательный уровень уводит его „вниз“ по энергии от точки пересечения с материнским дублетом, препятствуя тем самым его возвращению в дублет, откуда он быстро распался бы. В итоге ион-квартет лишается этого канала распада. Согласно расчетам B3LYP/6-311 + G**, два других канала его распада - в триплет и молекулу основного электронного состояния с инверсией спина, также невозможны, поскольку оба этих состояния нейтрали лежат по энергии выше ионаквартета. В итоге ион становится вечноживущим. Таким образом, первый пучок ионов $\mathrm{DQ}^{-}$, выходящих из IC, зарегистрированный в момент времени $t_{\mathrm{ext}}$, состоит именно из таких, вечноживущих ионов.

В то же время величина $\tau_{a}$ в процессе ее измерения регистрируется как конечная величина. Это значит, что в другой (более поздний) момент, а именно в момент измерения времени жизни ионов, из IC вместе с вечноживущими ионами ${ }^{\infty} \mathrm{M}^{-}$выходят также и временноживущие ${ }^{a} \mathrm{M}^{-}$, и, следовательно, не все ионы, достигающие системы регистрации при измерении $\tau_{a}$, проходят через адсорбцию. Этот факт подтверждается специальным экспериментом, проведенным в настоящей работе, когда были измерены величины $t_{\mathrm{ext}}$ для ионов $\mathrm{M}^{-}$и для нейтральной компоненты $\mathrm{M}^{0}$ отдельно. В отличие от $t_{\mathrm{ext}}\left(\mathrm{M}^{-}\right)$величина $t_{\mathrm{ext}}\left(\mathrm{M}^{0}\right)$ измерялась, когда на пластины конденсатора, используемого при измерении $\tau_{a}$, изначально подавался отрицательный потенциал с целью выделения из полного пучка нейтральных молекул, образующихся в результате автоотщепления электронов от ионов. Многократные измерения (и не только для DQ) продемонстрировали полную воспроизводимость результата и показали, что всегда $t_{\mathrm{ext}}\left(\mathrm{M}^{0}\right) \gg t_{\mathrm{ext}}\left(\mathrm{M}^{-}\right)$. Например, одно из измерений для DQ дает: $t_{\text {ext }}\left(\mathrm{M}^{-}\right)=5.1 \mu \mathrm{s}$ и $t_{\text {ext }}\left(\mathrm{M}^{0}\right)=61.35 \mu \mathrm{s}$, т.е. разница составляет очень большую величину $\sim 56 \mu \mathrm{s}$. Это означает, что, когда электронный луч открывается, поначалу все образующиеся ионы адсорбируются на поверхности IC, и это происходит до тех пор, пока их количество на ней не достигнет некоторого критического значения. Затем под действием кулоновского отталкивания они начинают вытеснять друг друга с поверхности. Именно этот момент регистрируется как первый приход ионов на SEM, т. е. как время $t_{\mathrm{ext}}$. При этом нейтральной компоненты в ионном пучке нет. Зарегистрированное время относится только к ионам (вечноживущим) и потому его можно обозначить как $t_{\mathrm{ext}}\left(\mathrm{M}^{-}\right)$. В то же время процесс образования новых ионов в IC продолжается, и на ее поверхности, очевидно, еще остаются свободные места, в результате чего вновь образующиеся ионы попрежнему продолжают садиться на нее. Однако через какое-то время $\left[t_{\text {ext }}\left(\mathrm{M}^{0}\right)-t_{\mathrm{ext}}\left(\mathrm{M}^{-}\right)\right]$вакантные места на поверхности заканчиваются и вновь образующиеся ионы уже больше не могут сесть на нее. Тогда они начинают свободно вылетать из IC, уже не превращаясь в вечноживущие ${ }^{\infty} \mathrm{M}^{-}$. Теперь общий ионный поток представляет собой смесь ${ }^{\infty} \mathrm{M}^{-}{ }_{\text {и }}{ }^{a} \mathrm{M}^{-}$и в нем уже есть нейтральная компонента $\left(\mathrm{M}^{0}\right)$, которая и регистрируется через время $t_{\mathrm{ext}}\left(\mathrm{M}^{0}\right)$ после открывания электронного луча. В итоге устанавливается динамическое равновесие между выходящими из камеры ионами двух типов: ${ }^{\infty} \mathrm{M}^{-}$ и ${ }^{a} \mathrm{M}^{-}$, смесь которых регистрируется в процессе измерения величины $\tau_{a}$. Очевидно, что вклад вечноживущих ионов $\infty^{-} \mathrm{M}^{-}$в общий ионный поток влечет за собой завышение измеряемой величины $\tau_{a}$. При этом доля ионов ${ }^{\infty} \mathrm{M}^{-}$зависит от многих факторов и потому результат измерения $\tau_{a}$ становится в определенной степени непредсказуемым, порождая разброс в измеряемой величине. Это означает, что именно эффект адсорбции ионов на стенках IC и появление в общем ионном пучке вечноживущей компоненты и является серьезной причиной существующего разброса экспериментально измеряемой величины $\tau_{a}$. Следует отметить, что подобное явление, т.е. регистрацию двух типов ионов со значительно отличающимися временами жизни, наблюдали также и авторы работы [3], что подтверждает результат настоящей работы и указывает на его универсальный характер. В работе [19] отмечался еще один эффект, который также может оказывать влияние на измерение времени жизни отрицательных ионов. Он относится к условиям в трубе анализатора масс-спектрометра и не связан с процессами, протекающими в IC. По-видимому, 
в ряде случаев он также должен учитываться наряду с эффектами, выявленными в настоящей работе.

С наличием в IC ионов двух типов ${ }^{\infty} \mathrm{M}^{-}$и ${ }^{a} \mathrm{M}^{-}$ тесно связан и характер измеряемой зависимости $\tau_{a}$ от температуры $(T)$ (рис. 3). Температурная зависимость $\tau_{a}=f(T)$ также искажается в результате адсорбции ионов в IC. Это видно из анализа изменений интенсивности ионов, которые наблюдаются при увеличении температуры IC. Интенсивность ионов играет ключевую роль в измерении $\tau_{a}$, поскольку время жизни определяется соотношением интенсивностей ионной и нейтральной компонент (см. (2)). И, как уже отмечалось выше, рис. 3 показывает, что с ростом температуры IC от 333 до $473 \mathrm{~K}$ величина $\tau_{a}$ уменьшается, и при этом интенсивность ионного пучка падает в 2.3 раза c $9 \cdot 10^{4}$ до $4 \cdot 10^{4} \mathrm{pulses} / \mathrm{s}$. Сама по себе величина $\tau_{a}$ конечна и должна падать с ростом температуры. Однако одновременное падение интенсивности, причем такое резкое, говорит о том, что эксперимент не является чистым. Это падение интенсивности ионов невозможно объяснить изменениями в заселенности колебательных уровней молекул при повышении температуры и соответствующим уменьшением временем жизни $\left(\tau_{a}\right)$ ионов, образующихся из колебательно возбужденных молекул, даже если предположить, что уменьшение $\tau_{a}$ этих возбужденных ионов настолько значительно, что они уже практически не долетают до системы регистрации. Невозможно объяснить потому, что число молекул, находящихся на нулевом колебательном уровне, при повышении температуры от 333 до $473 \mathrm{~K}$ не может уменьшиться в 2.3 раза. Действительно, в соответствии с распределением Больцмана, соотношение числа молекул на первом возбужденном колебательном уровне $\left(N_{1}\right)$ и на нулевом $\left(N_{0}\right)$ составляет

$$
N_{1} / N_{0}=\exp (-\Delta E / k T),
$$

где $k=8.6112 \cdot 10^{-5} \mathrm{eV} / \mathrm{K}$ - постоянная Больцмана, $\Delta E$ - энергия первого возбужденного колебательного уровня, $T$ - температура в $\mathrm{K}$. Для $\mathrm{DQ} \Delta E=$ $=0.0718 \mathrm{eV}\left(587 \mathrm{~cm}^{-1}\right)$ [20]. Из этого уравнения для $T_{1}=333 \mathrm{~K}$ следует $\left(N_{1} / N_{0}\right)^{\prime}=0.0818$, а для $T_{2}=473 \mathrm{~K}$ из этого же уравнения вытекает $\left(N_{1} / N_{0}\right)^{\prime \prime}=0.1675$. Поскольку общее число молекул сохраняется, $\left(N_{1}+N_{0}\right)^{\prime}=$ $=\left(N_{1}+N_{0}\right)^{\prime \prime}$, и тогда $N_{0}^{\prime} / N_{0}^{\prime \prime}=1.1$. Иными словами, с повышением температуры IC от 333 до $473 \mathrm{~K}$ заселенность нулевого колебательного уровня уменьшается в 1.1, но не в 2.3 раза. Следовательно, с ростом температуры интенсивность ионов $\mathrm{DQ}^{-}$падает не только (и, видимо, не столько) из-за увеличения заселенности возбужденных колебательных уровней исходных молекул и соответствующего уменьшения $\tau_{a}$ ионов, сколько из-за уменьшения в общем ионном пучке доли ионов ${ }^{\infty} \mathrm{M}^{-}$. А такое уменьшение доли ${ }^{\infty} \mathrm{M}^{-}$очень просто объясняется тем, что с ростом температуры толщина $L$ молекулярного слоя, покрывающего поверхность стенок IC, уменьшается, что приводит, в свою очередь, к увеличению вероятности туннелирования добавочного электрона с иона ${ }^{\infty} \mathrm{M}^{-}$на поверхность, т. е. к его гибели (нейтрализации). Таким образом, отсюда видно, каким именно образом адсорбция ионов на стенках IC вносит свой негативный вклад и в ту часть эксперимента, которая связана с изучением температурных зависимостей.

Следует отметить, что нейтрализация иона на поверхности IC возможна только при определенном соотношении энергетических характеристик иона и зонной структуры поверхности IC (нержавеющая сталь, покрытая сажей), когда добавочный электрон иона находится на одном энергетическом уровне с областью зоны проводимости. Наши оценки показывают, что в случае $\mathrm{DQ}^{-}$ это требование выполняется. Оценки были проведены с учетом стабилизация иона и соответственно его уровней, под воздействием положительного потенциала IC, который обычно приложен к IC [21], и индуцированного заряда на проводящей поверхности IC [22]. Аналогичные оценки были проведены для иона $\mathrm{Cl}^{-}$, которые показали, что для него туннелирование добавочного электрона запрещено (уровень с добавочным электроном иона $\mathrm{Cl}^{-}$попадает в область валентной зоны поверхности, т.е. в область занятых уровней). Это подтверждается и экспериментом, проведенным в настоящей работе с ионами $\mathrm{Cl}^{-}$из трихлорэтилена, интенсивность которых, как было экспериментально установлено, не падает с ростом температуры. Полученные результаты согласуются с данными других авторов. В частности, аналогичная роль толщины промежуточного слоя (н-гексан и $\mathrm{Kr}$ ) и зонной структуры в нейтрализации отрицательных ионов на поверхности $\mathrm{Pt}$ показана в работе [23].

Промежуточный слой молекул на поверхности IC

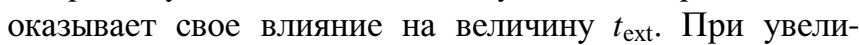
чении напуска вещества $L$ возрастает, связь иона с индуцированным зарядом ослабевает и $t_{\text {ехt }}$ уменьшается (рис. $2, b)$. Другой эффект наблюдается при изменении температуры IC. С ростом температуры толщина слоя $L$ уменьшается, связь иона с индуцированным зарядом укрепляется и $t_{\text {ext }}$ растет (рис. $3, b$ ).

В то же время можно видеть, что в обоих экспериментах и с напуском вещества, и с изменениями температуры, величина $\tau_{a}$ не коррелирует с $t_{\text {ext. }}$. Это происходит потому, что $\tau_{a}$ напрямую зависит от количественного соотношения ${ }^{\infty} \mathrm{M}^{-}$и ${ }^{a} \mathrm{M}^{-}$, но не от $t_{\text {ext }}$. Само соотношение ионов двух типов $\left({ }^{\infty} \mathrm{M}^{-}\right.$и $\left.{ }^{a} \mathrm{M}^{-}\right)$в

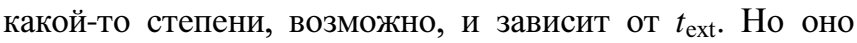
зависит также и от других факторов. Среди таковых большую роль играет площадь стенок IC, адсорбирующих ионы. Чем она больше, чем больше образуется ионов ${ }^{\infty} \mathrm{M}^{-}$. На количество адсорбированных ионов безусловно должны влиять также и физико-химические характеристики сажи, молекулярного слоя, его толщина и потенциалы на линзах. Сочетание всех этих факторов в итоге создают то или иное соотношение ${ }^{\infty} \mathrm{M}^{-}$и ${ }^{a} \mathrm{M}^{-}$, которое и определит в итоге зарегистрированную величину $\tau_{a}$. 


\section{Выводы}

Измерено время вытягивания отрицательных ионов из IC статического масс-спектрометра МИ-1201B, которое, существенно превышая время свободного вылета ионов из IC, указывает на адсорбцию части ионов на ее поверхности.

Показано, что ионы, сформировавшиеся первоначально как обычные временноживущие отрицательные молекулярные ионы, в результате адсорбции трансформируются в вечноживущие. Вклад последних в общий ионный пучок является главной причиной известной проблемы, которая возникает при экспериментальном измерении времени жизни отрицательных ионов и выражается в разбросе получаемых величин.

Установлено, что падение времени жизни ионов при повышении температуры IC обусловлено не только (или не столько) изменением заселенности колебательных уровней материнских молекул, сколько уменьшением доли вечноживущих ионов в общем ионном пучке. Уменьшение доли вечноживущих ионов при повышении температуры происходит из-за их более эффективной нейтрализации на поверхности IC в процессе туннелирования добавочного электрона с иона в проводящую поверхность IC, где более эффективное туннелирование обусловлено уменьшением толщины промежуточного молекулярного слоя, который отделяет ион от проводящей поверхности и принимает участие в формировании туннельного барьера.

\section{Список литературы}

[1] Илленбергер Е., Смирнов Б.М. // УФН. 1998. Т. 168. № 7. C. 731-766.

[2] Лукин В.Г., Воробьев А.С., Ломакин Г.С. // ПТЭ. 2009. № 4. C. $121-126$.

[3] Cannon M., Liu Y., Suess L., Dunning F.B., Steill J.D., Compton R.N. // J. Phys. Chem. 2007. Vol. 127. N 6. P. 064314(1-10).

[4] Барзилович П.П., Захарченко В.В., Зенкин В.И., Савин O.P., Шкордода В.Ф. // Приборы и системы управления. 1971. № 1. С. 24.

[5] Савин О.Р., Симоновский В.И., Стародуб Н.И. и др. // Приборы и системы управления. 1983. № 10. С. 13.

[6] Хвостенко В.И., Мазунов В.А., Фалько В.С. и др. // Хим. физика. 1982. № 7. С. 915-921.

[7] Лукин В.Г., Ломакин Г.С. // ПТЭ. 2010. № 3. С. 88-90.

[8] Устройство интерфейсное (техническое описание и инструкция по эксплуатации), Черноголовка. 1989.

[9] Трохов С.Е., Щербинин В.П., Сероклин Ю.Г., Некрасов Ю.С. // ПТЭ. 1975. № 3. С. 255.

[10] Хвостенко В.И., Фурлей И.И., Мазунов В.А., Костяновский Р.Г. // Изв. АН СССР. Сер. хим. 1973. Вып. 3. C. $680-681$.

[11] Лукин В.Г., Фалько В.С., Вязовкин Д.Е. // Автометрия. 1992. № 1. C. $111-115$.

[12] Руководство по эксплуатации цифрового осциллографа серии GDS-820C. М. 2007.
[13] De Boer J.H. The Dynamical Character of Adsorption. Oxford: Clarendon Press, 1968. 240 p.

[14] Khvostenko V.I., Tolstikov G.A. // Rus. Chem. Rev. 1976. Vol. 45. P. 127-141.

[15] Khvostenko O.G., Tuimedov G.M. // Rapid Comm. Mass Spectrom. 2006. N 20. P. 3699-3708.

[16] Хвостенко О.Г., Туймедов Г.М., Джемилев У.М. // Докл. AH CCCP. 2007. T. 414. № 6. C. 784-787.

[17] Khvostenko O.G., Shchukin P.V., Tuimedov G.M., Muftakhov M.V., Tseplin E.E., Tseplina S.N., Mazunov V.A. // Int. J. Mass Spectrom. 2008. Vol. 273. P. 69.

[18] Khvostenko O.G., Lukin V.G., Tseplin E.E. // Rapid Comm. Mass Spectrom. 2012. Vol. 26. P. 2535-2547.

[19] Туктаров Р.Ф., Хатымов Р.В., Щукин П.В. и др. // Письма в ЖЭТФ. 2009. Т. 90. Вып. 7. С. 564-568.

[20] NIST/ERA Gas — Phase Infrared Database.

[21] Лукин В.Г., Туймедов Г.М. // ПТЭ. 2013. N 5. С. 77-80.

[22] Sanche L. // J. Phys. B. At. Mol. Opt. 1990. Vol. 23. P. 1597-1624.

[23] Waddill G.D., Kesmodel L.L. // Phys. Rev. B. 1985. Vol. 32. P. 2107-2114. 